\title{
The First Mean Value Theorem for Integrals
}

\author{
Keiko Narita \\ Hirosaki-city \\ Aomori, Japan
}

\author{
Noboru Endou \\ Gifu National College of Technology \\ Japan \\ Yasunari Shidama \\ Shinshu University \\ Nagano, Japan
}

Summary. In this article, we prove the first mean value theorem for integrals [16]. The formalization of various theorems about the properties of the Lebesgue integral is also presented.

MML identifier: MESFUNC7, version: $\underline{7.8 .09 \quad 4.97 .1001}$

The notation and terminology used in this paper are introduced in the following articles: [20], [2], [17], [6], [1], [4], [21], [22], [11], [3], [9], [8], [10], [18], [19], [5], [13], [12], [14], [15], and [7].

\section{Lemmas for Extended Real Valued Functions}

For simplicity, we use the following convention: $X$ is a non empty set, $S$ is a $\sigma$-field of subsets of $X, M$ is a $\sigma$-measure on $S, f, g$ are partial functions from $X$ to $\overline{\mathbb{R}}$, and $E$ is an element of $S$.

One can prove the following three propositions:

(1) If for every element $x$ of $X$ such that $x \in \operatorname{dom} f$ holds $f(x) \leq g(x)$, then $g-f$ is non-negative.

(2) For every set $Y$ and for every partial function $f$ from $X$ to $\overline{\mathbb{R}}$ and for every real number $r$ holds $(r f)\lceil Y=r(f\lceil Y)$.

(3) Suppose $f$ is integrable on $M$ and $g$ is integrable on $M$ and $g-f$ is nonnegative. Then there exists an element $E$ of $S$ such that $E=\operatorname{dom} f \cap \operatorname{dom} g$ and $\int f\left\lceil E \mathrm{~d} M \leq \int g\lceil E \mathrm{~d} M\right.$. 


\section{2. $\sigma$-Finite SETS}

Let us consider $X$. One can verify that there exists a partial function from $X$ to $\overline{\mathbb{R}}$ which is non-negative. $\overline{\mathbb{R}}$.

Let us consider $X, f$. Then $|f|$ is a non-negative partial function from $X$ to

Next we state the proposition

(4) Suppose $f$ is integrable on $M$. Then there exists a function $F$ from $\mathbb{N}$ into $S$ such that

(i) for every element $n$ of $\mathbb{N}$ holds $F(n)=\operatorname{dom} f \cap \operatorname{GTE}-\operatorname{dom}\left(|f|, \overline{\mathbb{R}}\left(\frac{1}{n+1}\right)\right)$,

(ii) $\operatorname{dom} f \backslash \mathrm{EQ}-\operatorname{dom}\left(f, 0_{\overline{\mathbb{R}}}\right)=\bigcup \operatorname{rng} F$, and

(iii) for every element $n$ of $\mathbb{N}$ holds $F(n) \in S$ and $M(F(n))<+\infty$.

\section{The First Mean Value Theorem for Integrals}

Let $F$ be a binary relation. We introduce $F$ is extreal-yielding as a synonym of $F$ is extended real-valued.

Let $k$ be a natural number and let $x$ be an element of $\overline{\mathbb{R}}$. Then $k \mapsto x$ is a finite sequence of elements of $\overline{\mathbb{R}}$.

Let us note that there exists a finite sequence which is extreal-yielding.

The binary operation $\cdot \overline{\mathbb{R}}$ on $\overline{\mathbb{R}}$ is defined by:

$(\text { Def. } 2)^{1} \quad$ For all elements $x, y$ of $\overline{\mathbb{R}}$ holds $\cdot \overline{\mathbb{R}}(x, y)=x \cdot y$.

One can check that $\cdot \overline{\mathbb{R}}$ is commutative and associative.

One can prove the following proposition

(5) $\mathbf{1}_{\cdot_{\mathbb{R}}}=1$.

One can check that $\cdot \overline{\mathbb{R}}$ is unital.

Let $F$ be an extreal-yielding finite sequence. The functor $\prod F$ yields an element of $\overline{\mathbb{R}}$ and is defined by:

(Def. 3) There exists a finite sequence $f$ of elements of $\overline{\mathbb{R}}$ such that $f=F$ and $\prod F=\cdot \overline{\mathbb{R}} \circledast f$.

Let $x$ be an element of $\overline{\mathbb{R}}$ and let $n$ be a natural number. Note that $n \mapsto x$ is extreal-yielding.

Let $x$ be an element of $\overline{\mathbb{R}}$ and let $k$ be a natural number. The functor $x^{k}$ is defined by:

(Def. 4) $\quad x^{k}=\prod(k \mapsto x)$.

Let $x$ be an element of $\overline{\mathbb{R}}$ and let $k$ be a natural number. Then $x^{k}$ is an extended real number.

Let us note that $\varepsilon_{\overline{\mathbb{R}}}$ is extreal-yielding.

\footnotetext{
${ }^{1}$ The definition (Def. 1) has been removed.
} 
Let $r$ be an element of $\overline{\mathbb{R}}$. Note that $\langle r\rangle$ is extreal-yielding.

We now state two propositions:

(6) $\prod\left(\varepsilon_{\overline{\mathbb{R}}}\right)=1$.

(7) For every element $r$ of $\overline{\mathbb{R}}$ holds $\prod\langle r\rangle=r$.

Let $f, g$ be extreal-yielding finite sequences. Observe that $f \frown g$ is extrealyielding.

We now state three propositions:

(8) For every extreal-yielding finite sequence $F$ and for every element $r$ of $\overline{\mathbb{R}}$ holds $\prod\left(F^{\frown}\langle r\rangle\right)=\prod F \cdot r$.

(9) For every element $x$ of $\overline{\mathbb{R}}$ holds $x^{1}=x$.

(10) For every element $x$ of $\overline{\mathbb{R}}$ and for every natural number $k$ holds $x^{k+1}=$ $x^{k} \cdot x$.

Let $k$ be a natural number and let us consider $X, f$. The functor $f^{k}$ yields a partial function from $X$ to $\overline{\mathbb{R}}$ and is defined by:

(Def. 5) $\operatorname{dom}\left(f^{k}\right)=\operatorname{dom} f$ and for every element $x$ of $X$ such that $x \in \operatorname{dom}\left(f^{k}\right)$ holds $f^{k}(x)=f(x)^{k}$.

Next we state several propositions:

(11) For every element $x$ of $\overline{\mathbb{R}}$ and for every real number $y$ and for every natural number $k$ such that $x=y$ holds $x^{k}=y^{k}$.

(12) For every element $x$ of $\overline{\mathbb{R}}$ and for every natural number $k$ such that $0 \leq x$ holds $0 \leq x^{k}$.

(13) For every natural number $k$ such that $1 \leq k$ holds $+\infty^{k}=+\infty$.

(14) Let $k$ be a natural number and given $X, S, f, E$. If $E \subseteq \operatorname{dom} f$ and $f$ is measurable on $E$, then $|f|^{k}$ is measurable on $E$.

(15) Suppose $\operatorname{dom} f \cap \operatorname{dom} g=E$ and $f$ is finite and $g$ is finite and $f$ is measurable on $E$ and $g$ is measurable on $E$. Then $f g$ is measurable on $E$.

(16) If $\operatorname{rng} f$ is bounded, then $f$ is finite.

(17) Let $M$ be a $\sigma$-measure on $S, f, g$ be partial functions from $X$ to $\overline{\mathbb{R}}, E$ be an element of $S$, and $F$ be a non empty subset of $\overline{\mathbb{R}}$. Suppose $\operatorname{dom} f \cap$ $\operatorname{dom} g=E$ and $\operatorname{rng} f=F$ and $g$ is finite and $f$ is measurable on $E$ and $\operatorname{rng} f$ is bounded and $g$ is integrable on $M$. Then $(f g)\lceil E$ is integrable on $M$ and there exists an element $c$ of $\mathbb{R}$ such that $c \geq \inf F$ and $c \leq \sup F$ and $\int(f|g|)\left\lceil E \mathrm{~d} M=\overline{\mathbb{R}}(c) \cdot \int|g|\lceil E \mathrm{~d} M\right.$.

\section{Selected Properties of Integrals}

We use the following convention: $E_{1}, E_{2}$ denote elements of $S, x, A$ denote sets, and $a, b$ denote real numbers.

The following propositions are true: 
(18) $\quad|f|\lceil A=\mid f\lceil A \mid$.

(19) $\operatorname{dom}(|f|+|g|)=\operatorname{dom} f \cap \operatorname{dom} g$ and $\operatorname{dom}|f+g| \subseteq \operatorname{dom}|f|$.

(20) $\quad|f|\lceil\operatorname{dom}|f+g|+|g| \uparrow \operatorname{dom}|f+g|=(|f|+|g|)\lceil\operatorname{dom}|f+g|$.

(21) If $x \in \operatorname{dom}|f+g|$, then $|f+g|(x) \leq(|f|+|g|)(x)$.

(22) Suppose $f$ is integrable on $M$ and $g$ is integrable on $M$. Then there exists an element $E$ of $S$ such that $E=\operatorname{dom}(f+g)$ and $\int|f+g|\lceil E \mathrm{~d} M \leq$ $\int|f|\left\lceil E \mathrm{~d} M+\int|g| \mid E \mathrm{~d} M\right.$.

(23) $\max _{+}\left(\chi_{A, X}\right)=\chi_{A, X}$.

(24) If $M(E)<+\infty$, then $\chi_{E, X}$ is integrable on $M$ and $\int \chi_{E, X} \mathrm{~d} M=M(E)$ and $\int \chi_{E, X}\lceil E \mathrm{~d} M=M(E)$.

(25) If $M\left(E_{1} \cap E_{2}\right)<+\infty$, then $\int \chi_{\left(E_{1}\right), X}\left\lceil E_{2} \mathrm{~d} M=M\left(E_{1} \cap E_{2}\right)\right.$.

(26) Suppose $f$ is integrable on $M$ and $E \subseteq \operatorname{dom} f$ and $M(E)<+\infty$ and for every element $x$ of $X$ such that $x \in E$ holds $a \leq f(x) \leq b$. Then $\overline{\mathbb{R}}(a) \cdot M(E) \leq \int f\lceil E \mathrm{~d} M \leq \overline{\mathbb{R}}(b) \cdot M(E)$.

\section{REFERENCES}

[1] Grzegorz Bancerek. The fundamental properties of natural numbers. Formalized Mathematics, 1(1):41-46, 1990.

[2] Grzegorz Bancerek. The ordinal numbers. Formalized Mathematics, 1(1):91-96, 1990.

[3] Grzegorz Bancerek and Krzysztof Hryniewiecki. Segments of natural numbers and finite sequences. Formalized Mathematics, 1(1):107-114, 1990.

[4] Józef Białas. Infimum and supremum of the set of real numbers. Measure theory. Formalized Mathematics, 2(1):163-171, 1991.

[5] Józef Białas. Series of positive real numbers. Measure theory. Formalized Mathematics, 2(1):173-183, 1991

[6] Józef Białas. The $\sigma$-additive measure theory. Formalized Mathematics, 2(2):263-270, 1991

[7] Józef Białas. Some properties of the intervals. Formalized Mathematics, 5(1):21-26, 1996.

[8] Czesław Byliński. Binary operations. Formalized Mathematics, 1(1):175-180, 1990.

[9] Czesław Byliński. Finite sequences and tuples of elements of a non-empty sets. Formalized Mathematics, 1(3):529-536, 1990.

[10] Czesław Byliński. Functions from a set to a set. Formalized Mathematics, 1(1):153-164, 1990.

[11] Czesław Byliński. Partial functions. Formalized Mathematics, 1(2):357-367, 1990.

[12] Noboru Endou and Yasunari Shidama. Integral of measurable function. Formalized Mathematics, 14(2):53-70, 2006.

[13] Noboru Endou, Katsumi Wasaki, and Yasunari Shidama. Basic properties of extended real numbers. Formalized Mathematics, 9(3):491-494, 2001.

[14] Noboru Endou, Katsumi Wasaki, and Yasunari Shidama. Definitions and basic properties of measurable functions. Formalized Mathematics, 9(3):495-500, 2001.

[15] Noboru Endou, Katsumi Wasaki, and Yasunari Shidama. The measurability of extended real valued functions. Formalized Mathematics, 9(3):525-529, 2001.

[16] P. R. Halmos. Measure Theory. Springer-Verlag, 1987.

[17] Krzysztof Hryniewiecki. Basic properties of real numbers. Formalized Mathematics, 1(1):35-40, 1990.

[18] Rafał Kwiatek. Factorial and Newton coefficients. Formalized Mathematics, 1(5):887-890, 1990.

[19] Andrzej Nędzusiak. $\sigma$-fields and probability. Formalized Mathematics, 1(2):401-407, 1990.

[20] Zinaida Trybulec. Properties of subsets. Formalized Mathematics, 1(1):67-71, 1990.

[21] Edmund Woronowicz. Relations and their basic properties. Formalized Mathematics, 1(1):73-83, 1990. 
[22] Edmund Woronowicz. Relations defined on sets. Formalized Mathematics, 1(1):181-186, 1990.

Received October 30, 2007 